\title{
Effects of Nerve Growth Factor on the Survival and Synaptic Function of la Sensory Neurons Axotomized in Neonatal Rats
}

\author{
Y. Miyata, Y. Kashihara, S. Homma, and M. Kuno \\ National Institute for Physiological Sciences, Okazaki 444, Japan
}

Sensory neurons with small diameters (A $\delta$ and $C$ cells) are known to be responsive to exogenous NGF even at postnatal stages. We have examined whether large Group Ia sensory neurons (A $\alpha$ cells) arising from muscle spindles are also responsive to NGF in neonatal rats. For this purpose, monosynaptic excitatory postsynaptic potentials (EPSPs) were evoked in spinal motoneurons by Group Ia muscle afferent volleys. When a muscle nerve was crushed on the day after birth, the monosynaptic EPSPs elicited by afferent volleys from the muscle were depressed within several weeks. This synaptic depression was partially reversed by daily treatment with NGF. NGF treatment also enhanced the EPSPs evoked by stimulation of intact muscle nerves, but this effect was less marked than that on the EPSPs produced by stimulation of the previously crushed muscle nerve. Exogenous NGF was effective for the EPSPs when the treatment began on the day after birth but not when the treatment began $4 \mathrm{~d}$ after birth. Following crush of a muscle nerve on the day after birth, about $\mathbf{4 5 \%}$ of the sensory neurons derived from the muscle were lost. The cell death of small sensory neurons was prevented by daily treatment with NGF, whereas the NGF treatment was ineffective in preventing the cell death of large sensory neurons. The results indicate that Group Ia sensory neurons are responsive to NGF during early postnatal life.

Group Ia sensory fibers arising from a muscle form excitatory synapses on spinal motoneurons that innervate the muscle and its synergists (Eccles, 1957). When a muscle nerve is sectioned or crushed in adult cats, monosynaptic excitatory postsynaptic potentials (EPSPs) evoked in spinal motoneurons by afferent volleys from the muscle are significantly depressed within 2 weeks (Eccles et al., 1959; Gallego et al., 1979, 1980; Goldring et al., 1980). The EPSPs once depressed recover to near normal levels or even become enhanced as regeneration of the peripheral process occurs 4-5 weeks after the nerve crush (Gallego et al., 1980; Goldring et al., 1980). However, the recovery of EPSPs does not require functional restoration of the peripheral sensory response to muscle stretch (Goldring et al., 1980). This raises the possibility that the recovery of central synaptic function is triggered by some trophic factor supplied by contact of the peripheral sensory terminals with the muscle, rather than by sensory impulse activity.

Recently, NGF has been shown to play a trophic role for sensory neurons early in postnatal or even adult animals. Sensory fibers retrogradely transport NGF throughout life (Stöckel

Received Oct. 7, 1985; revised Dec. 9, 1985; accepted Feb. 14, 1986.

We wish to thank Dr. Noboru Mizuno and his colleagues for their pertinent advice concerning HRP staining techniques and Dr. Masaharu Ogawa for detailed instruction in preparing NGF and its antiserum. We are also grateful to Drs. Shinichiro Hori and Kyozo Hayashi for providing us with purified NGF and its antiserum in an early stage of this study.

Correspondence should be addressed to M. Kuno, Department of Physiology, Kyoto University Faculty of Medicine, Kyoto 606, Japan.

Copyright (c) 1986 Society for Neuroscience $0270-6474 / 86 / 072012-07 \$ 02.00 / 0$ et al., 1975a, b). The substance $\mathrm{P}$ content in sensory neurons decreases following chronic section of the peripheral nerve, but this reduction can be prevented by daily administration of NGF (Fitzgerald et al., 1985; Goedert et al., 1981; Kessler and Black, 1980; Otten et al., 1980). Similarly, fluoride-resistant acid phosphatase in the dorsal horn, which is normally depleted after peripheral nerve section, can be maintained by NGF treatment (Csillik et al., 1985; Fitzgerald et al., 1985). Thus, the smalldiameter sensory neurons (A $\delta$ and $C$ cells), identified by their chemical markers, appear to use NGF as a trophic factor. However, it is not known whether this is true for large sensory neurons. While no adequate chemical marker is available for large Group Ia sensory neurons (A $\alpha$ cells), their central synaptic function can be quantified by the amplitude of monosynaptic EPSPs evoked in spinal motoneurons by stimulation of the Group Ia fibers. We have examined whether depression of the EPSPs produced by peripheral nerve crush may be affected by daily treatment with NGF in nconatal rats. Crush of the peripheral nerve in neonatal rats is known to cause massive cell death of sensory neurons (Bondok and Sansone, 1984; Yip and Johnson, 1984; Yip et al., 1984). The present results show that NGF treatment partially reverses the EPSP depression induced by peripheral nerve crush, whereas the treatment is incffective in preventing the cell death of large sensory neurons. A preliminary report on parts of this work has appeared (Kuno et al., 1985).

\section{Materials and Methods}

\section{Preparation}

Wistar rats were anesthetized with ether on the day after birth, and the nerve to the medial gastrocnemius (MG) muscle was exposed in the left hind leg. The MG nerve was found to have a bifurcation near the muscle. The nerve was crushed just central to the branching point with a Dumont forceps (No. 5). The wound was closed with 9-0 silk suture and the pups returned to their mother. In another group of animals, the nerve crush was made $4 \mathrm{~d}$ after birth. Each litter size was adjusted to 8 pups.

\section{Electrophysiological observations}

After a postoperative period of 24-35 d, the rat was anesthetized by an intraperitoneal injection of pentobarbital sodium $(50 \mathrm{mg} / \mathrm{kg})$. One-third the initial dose was subsequently given when the cornea reflex recovered. The spinal cord was transected at the midthoracic level. After tracheostomy, the lumbar cord was exposed by laminectomy, leaving all the dorsal and ventral roots intact. In the left hind leg, the MG and lateral gastrocnemius-soleus (LGS) nerves were exposed. Functional reinnervation of the muscle by the previously crushed MG nerve was invariably present. The MG and LGS nerves were cut distally and prepared for stimulation separately. Intracellular recording from spinal motoneurons was performed with glass microelectrodes filled with $2 \mathrm{M}$ potassium acetate. Satisfactory electrodes had resistances between 15 and $30 \mathrm{M} \Omega$. The fixation of the spinal cord was achieved by 2 spinous process clamps placed just above and below the laminectomy site and by a pair of horizontal metal clamps grasping the vertebral column near the recording site. Natural respiration was blocked by overventilation with an artificial respirator, and respiratory movements were minimized by pneumothorax. 
Intracellular recordings from MG or LGS motoneurons were identified by antidromic action potentials evoked by stimulation of the muscle nerve. Motoneurons with an action potential of less than $60 \mathrm{mV}$ were discarded. Monosynaptic EPSPs werc recorded in response to stimulation of each of the two muscle nerves at a frequency of $0.5 \mathrm{~Hz}$ at the intensity that yielded the maximum amplitude. Occasionally, the antidromic action potential was initiated at a stimulus intensity weaker than that required for the maximum homonymous EPSP. In such cases, the antidromic action potential was blocked to the $\mathrm{M}$-spike by applying hyperpolarizing currents through the intracellular electrode. Thus, it was possible to dissociate the homonymous EPSP from the antidromic M-spike, as reported previously in detail (Burke, 1968; Gallego et al., 1979). The applied hyperpolarizing current did not significantly affect the EPSP amplitude, as examined for the heteronymous EPSPs recorded from the same motoneuron (Gallego et al., 1979). The homonymous EPSP amplitude measured by this method might be $0.3-0.6 \mathrm{mV}$ larger than the true value (Hamm et al., 1983). No correction was made for this possible error since, in the majority of motoncurons cxamincd (about 80\%), the maximum homonymous EPSPs were obtained at stimulus intensities below the threshold for the motor axons. In response to stimulation of the previously crushed MG nerve, the EPSP amplitude often showed fluctuations from trial to trial. Therefore, the largest response among about 12 consecutively evoked EPSPs was used as the maximum EPSP.

\section{Morphological observations}

The possible cell death of sensory neurons following crush of the MG nerve was quantitatively evaluated by counting dorsal root ganglion (DRG) cells retrogradely labeled with HRP. Under pentobarbital anesthesia, the MG nerves were exposed on both the experimental and contralateral intact sides $24-35 \mathrm{~d}$ after the nerve crush operation. The MG nerves were freed of surrounding tissues and sectioned near the muscle. Immediately after the section, the central stump of the MG nerve was inserted into a plastic tube filled with $30 \%$ HRP (Toyobo Grade I-C) dissolved in $0.9 \%$ saline. Vaseline was applied to the tube orifice to prevent leakage of the solution. The nerve was left in the tube for $1 \mathrm{hr}$. After a survival time of $30-40 \mathrm{hr}$, the animal was anesthetized and perfused through the ascending aorta with $8 \%$ formalin in $0.1 \mathrm{M}$ phosphate buffer, $\mathrm{pH} 7.3$, followed by the same buffer containing $10 \%$ sucrose. The fourth (L4) and fifth (L5) lumbar DRG were bilaterally excised and stored in the phosphate buffer containing $25 \%$ sucrose. A few days later, each ganglion was embedded in $10 \%$ gelatin, and the gclatin block was placed in the buffer containing $1 \%$ glutaraldehyde and $25 \%$ sucrose overnight. Serial sections were cut at $50 \mu \mathrm{m}$ on a freezing microtome. The sections were treated with $3,3^{\prime}$-diaminobenzidine tetrahydrochloride and hydrogen peroxide (Streit and Reubi, 1977). After a washing in $0.9 \%$ saline, the sections were mounted in order on glass slides coated with alum-gelatin and counterstained with $1 \%$ cresyl violet. All the DRG neurons containing HRP-positive granules with a clear cell contour were counted. While the nucleus was clearly seen in small cells, it was not visible in many large cells. Thus, it is possible that the same cell might have been counted twice. To estimate the degree of this possible error, the position of every HRP-positive cell in each section was compared with that in adjacent sections in 2 animals (8 DRG). This revealed that about $10 \%$ of the HRP-positive cells counted spanned 2 neighboring sections. However, the smaller profiles of the cells found to span 2 neighboring sections were so small that they were usually excluded even by the regular counting. Consequently, the value estimated by the regular counting was very close $( \pm 2 \%)$ to that obtained after exclusion of the small profiles of the cells that spanned 2 neighboring sections.

\section{Preparation of NGF and anti-NGF antiserum}

The general procedures for preparing NGF and its antiserum have been described in a previous report (Kuno et al., 1985). According to the methods of Bocchini and Angeletti (1969), 2.5 S NGF was purified from the submaxillary glands of adult male mice. The $2.5 \mathrm{~S}$ NGF amounted to 250 biological units $/ \mu \mathrm{g}$ of protein. The NGF, $2 \mu \mathrm{g} / \mathrm{gm}$ body weight, was injected intraperitoneally daily for 1 week, starting on the day of the nerve crush operation. Thereafter, the same dose of NGF was administered every other day until the day before sacrifice. In order to observe the effectiveness of the NGF treatment, the superior cervical sympathetic ganglion (SCG) was exposed at the end of the experiment. In a few animals, the SCG was prepared for histological examination. All the NGF-treated animals showed a marked hypertrophy of the SCG
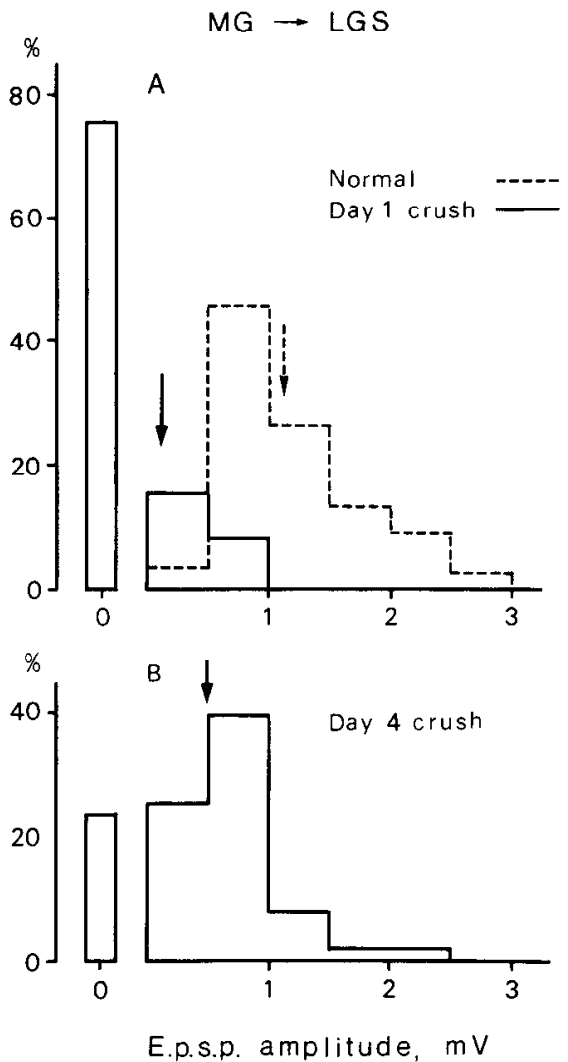

Figure 1. Amplitude distributions of monosynaptic EPSPs evoked in LGS motoneurons by stimulation of the MG nerve. Arrows indicate mean amplitudes. Each mean amplitude, its SD, and the numbers of motoneurons recorded $(n)$ follow. $A$, Results obtained from 8 rats whose MG nerves had been crushed the day after birth (solid-line histogram: $0.10 \pm 0.20 \mathrm{mV} ; n=58$ ) and those recorded from 20 age-matched unoperated animals (dashed-line histogram: $1.1 \pm 0.55 \mathrm{mV} ; n=146$ ). $B$, Results obtained from 10 rats whose MG nerves had been crushed $4 \mathrm{~d}$ after birth $(0.48 \pm 0.43 \mathrm{mV} ; n=63)$.

compared with age-matched (littermates) normal rats. Also, as notcd previously (Kornblum and Johnson, 1982), the body weight of the animals chronically treated with NGF was significantly lower than that of normal littermates.

The antiserum against NGF was prepared by injecting the $2.5 \mathrm{~S}$ mouse NGF into rabbits with complete Freund's adjuvant. The titer of the antiserum assayed by the chick embryo DRG (Fenton, 1970) was 600010,000 . The antiserum was injected subcutaneously daily for 1 week from the day after birth at a dose of $10 \mu \mathrm{l} / \mathrm{gm}$ body weight. After a 1 week pause, the same dose of the antiserum was administered again for 7 consecutive d. These animals showed a marked atrophy of the SCG.

The mean EPSP amplitudes and other motoneuron properties observed under different experimental conditions were examined by twotailed $t$ tests with the significance limit of $p<0.05$. The mean, its SD, and the sample size are given in the figure legends.

\section{Results}

\section{Effects of nerve crush on EPSPS}

Figure 1 shows the amplitude distributions of monosynaptic EPSPs produced in LGS motoneurons by stimulation of the MG nerve. In age-matched normal rats, the mean maximum amplitude of the heteronymous (from MG nerve to LGS motoneurons) EPSPs was $1.1 \mathrm{mV}$ (Fig. $1 A$, dashed-line histogram). In the animals whose $M G$ nerves had been crushed the day after birth, stimulation of the MG nerve did not elicit any monosynaptic EPSPs in $76 \%$ of the LGS motoneurons examined (Fig. $1 A$, solid-line histogram at 0 ). The mean amplitude of the EPSPs was only $0.1 \mathrm{mV}$. The degree of synaptic depression apparently 

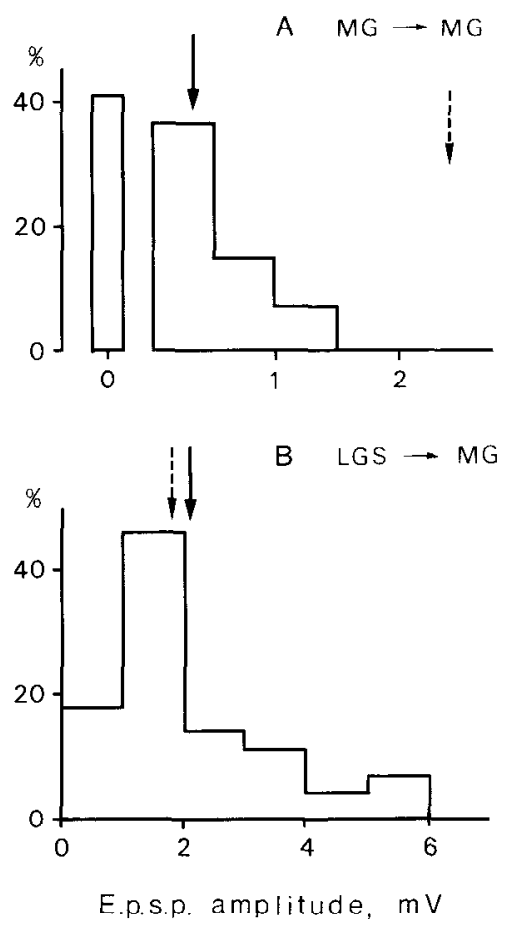

Figure 2. Amplitude distributions of monosynaptic EPSPs evoked in MG motoneurons in animals whose MG nerves had been crushed on the day after birth. Arrows indicate mean amplitudes. Their SDs and the numbers of motoneurons recorded $(n)$ follow. $A$, Homonymous EPSPs elicited by stimulation of the crushed MG nerve $(0.31 \pm 0.41$ $\mathrm{mV} ; n=28$ ); dashed-line arrow indicates the mean amplitude of the homonymous EPSPs recorded from age-matched unoperated rats $(2.4 \pm$ $0.90 \mathrm{mV} ; n=28$ ). $B$, Heteronymous EPSPs evoked by stimulation of the intact LGS nerve $(2.1 \pm 1.4 \mathrm{mV} ; n=28)$; dashed-line arrow indicates the mean amplitude of the heteronymous EPSPs recorded from unoperated rats $(1.8 \pm 0.69 \mathrm{mV} ; n=28)$.

depended upon the time of nerve crush after birth. As is shown in Figure $1 B$, in the animals whose MG nerves had been crushed $4 \mathrm{~d}$ after birth, stimulation of the crushed MG nerve failed to evoke EPSPs in only $24 \%$ of the LGS motoneurons recorded. The mean maximum EPSP amplitude $(0.5 \mathrm{mV}$; Fig. $1 B$, arrow) was significantly larger than that observed following nerve crush on day 1 (Fig. $1 A$, solid arrow) but significantly smaller than the control EPSPs (Fig. 1A, dashed-line arrow). Since these EPSPs were recorded from intact LGS motoneurons in response to stimulation of the crushed MG nerve, the observed synaptic depression appears to result from alterations of the MG Group Ia sensory fibers following the nerve crush.

Figure 2 shows the amplitude distributions of monosynaptic EPSPs recorded from MG motoneurons in response to stimulation of the MG $(A)$ or the LGS $(B)$ nerve after crush of the MG nerve on the day after birth. The mean amplitude of the MG homonymous EPSPs ( $0.3 \mathrm{mV}$; Fig. $2 A$, solid arrow) was only $13 \%$ of that observed in unoperated age-matched rats $(2.4$ $\mathrm{mV}$; dashed-line arrow). This was comparable to the degree of depression of the EPSPs evoked in intact LGS motoncurons by stimulation of the crushed MG nerve ( $9 \%$ of control; Fig. $1 A$ ). In contrast, the mean amplitude of the heteronymous EPSPs evoked by stimulation of the intact LGS nerve $(2.1 \mathrm{mV}$; Fig. $2 B$, solid arrow) was not significantly different from the control valuc $(1.8 \mathrm{mV}$; dashed-line arrow). Essentially the same results were observed following crush of the MG nerve $4 \mathrm{~d}$ after birth. The homonymous MG EPSP amplitude in these animals was significantly smaller, by about $33 \%$, than the normal value, whereas the heteronymous EPSPs evoked by stimulation of the
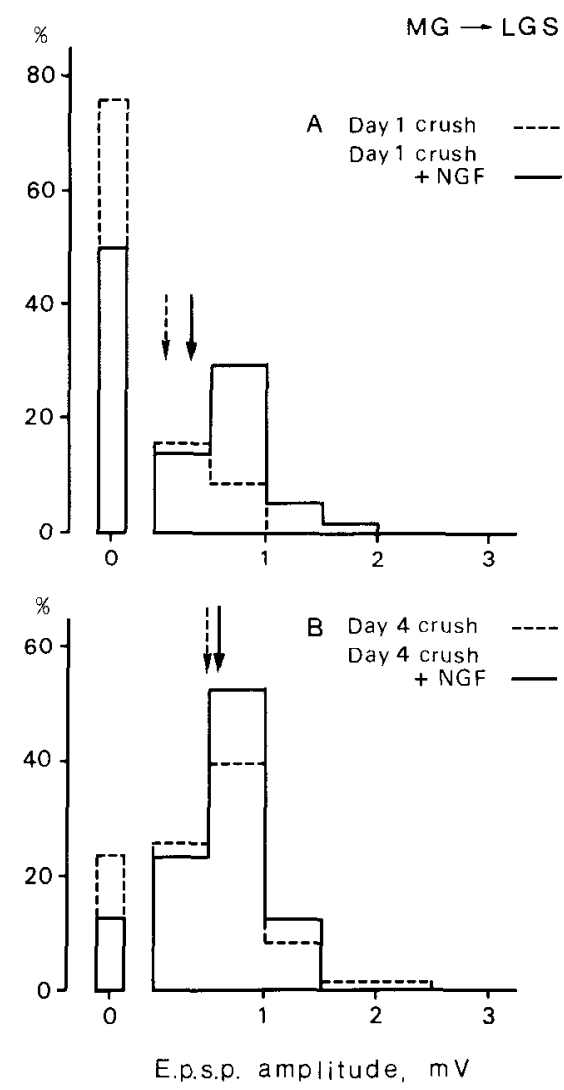

Figure 3. Effects of NGF on monosynaptic EPSPs. The EPSPs were recorded from intact LGS motoneurons in response to stimulation of the crushed MG nerve. Arrows indicate mean amplitudes. Their SDs and the numbers of motoneurons examined ( $n$ ) follow. $A$, Results obtained from the animals whose MG nerves had been crushed the day after birth (dashed-line histogram: reproduced from Fig. $2 A$ ) and those from 11 rats treated with NGF after the same operation (solid-line histogram: $0.33 \pm 0.38 \mathrm{mV} ; n=58$ ). $B$, Results obtained from animals whose MG nerves had been crushed $4 \mathrm{~d}$ after birth (dashed-line histogram; reproduced from Fig. $2 B$ ) and those from 9 rats treated with NGF after the same operation (solid-line histogram: $0.56 \pm 0.33 \mathrm{mV} ; n=$ 48).

intact LGS nerve (mean, $1.9 \mathrm{mV}$ ) were not significantly different from the normal value. Therefore, the synaptic depression observed following crush of the MG nerve appears to be confined to those synapses formed by the Group Ia sensory fibers that had been crushed.

\section{Effects of NGF on EPSPS}

In Figure $3 A$, the amplitude distribution of monosynaptic EPSPs evoked in intact LGS motoneurons by stimulation of the MG nerve that had been crushed the day after birth (dashed-line histogram) is compared to that observed in the animals treated with NGF immediately after the same operation (solid-line histogram). In the NGF-treated animals, the population of LGS motoneurons that showed no EPSPs to stimulation of the crushed MG ncrve was smaller than that found following the nerve crush alone. A threefold increase in the mean EPSP amplitude resulting from daily treatment with NGF (Fig. $3 A$, solid-line arrow) was statistically significant $(p<0.001)$. However, this reversal by NGF was still only partial, compared with the normal value (see Fig. 1A, dashed-line histogram). Figure $3 B$ shows the effects of NGF in the animals whose MG nerves had been crushed $4 \mathrm{~d}$ after birth. NGF treatment immediately after the nerve crush operation (solid-line histogram) had no significant effects on the EPSPs evoked in LGS motoneurons by stimulation 

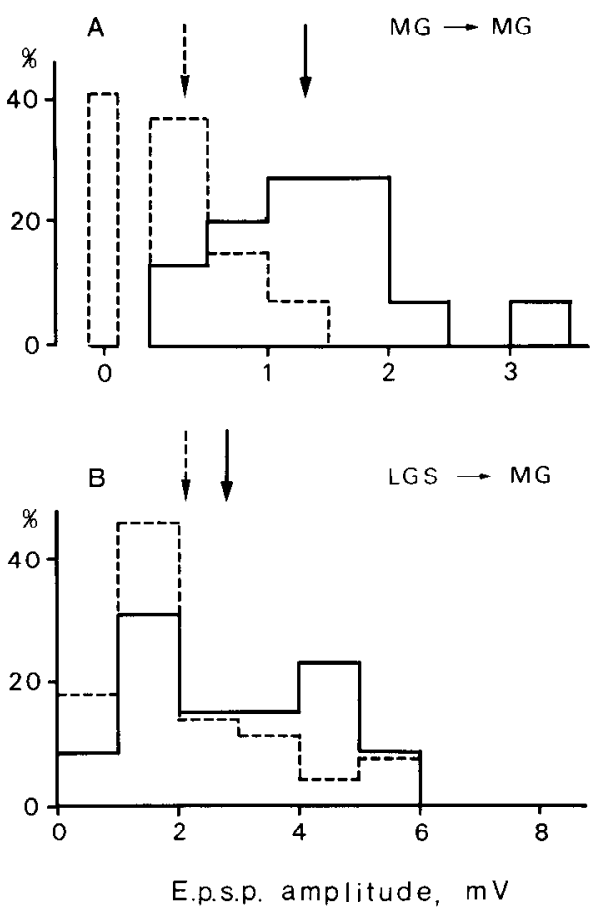

Figure 4. Effects of NGF on the EPSPs evoked by stimulation of the crushed MG nerve and by stimulation of the intact LGS nerve. The EPSP amplitude distributions were obtained from 8 animals whose MG nerves had been crushed the day after birth (dashed-line histograms) and from 11 rats treated with NGF after the same operation (solid-line histograms). Arrows indicate mean amplitudes. Their SDs and the numbers of motoneurons examined $(n)$ follow. All EPSPs were recorded from MG motoneurons. $A$. Homonymous EPSPs evoked by stimulation of the MG nerve. Results following MG nerve crush alone $(0.31 \pm 0.41$ $\mathrm{mV} ; n=28)$ and those with NGF treatment $(1.3 \pm 0.75 \mathrm{mV} ; n=15)$. $B$, Heteronymous EPSPs evoked by stimulation of the intact LGS nerve. Results following the MG nerve alone $(2.1 \pm 1.4 \mathrm{mV} ; n=28)$ and those with NGF treatment $(2.8 \pm 1.3 \mathrm{mV} ; n=15)$.

of the crushed MG nerve (dashed-line histogram). These results suggest that the EPSPs are responsive to exogenous NGF only when the treatment is made during early postnatal life.

Does the NGF treatment also enhance the EPSPs elicited by stimulation of the intact LGS nerve? Illustrated in Figure 4 are the amplitude distributions of monosynaptic EPSPs evoked in MG motoneurons by stimulation of the crushed MG nerve $(A)$ and by stimulation of the intact LGS nerve $(B)$. In these experiments, the MG nerve was crushed on the day after birth, and the results with NGF treatment (solid-line histograms) were compared to those following nerve crush alone (dashed-line histograms). The NGF treatment significantly $(p<0.001)$ increased the homonymous EPSPs evoked by stimulation of the crushed MG nerve (Fig. 4A). The mean EPSP amplitude in NGF-treated animals $(1.3 \mathrm{mV})$ was $4.3 \times$ larger than that following nerve crush alone $(0.3 \mathrm{mV}$; Fig. $4 A)$. However, the former value was still $54 \%$ of that observed in normal, unoperated rats $(2.4 \mathrm{mV})$. The NGF treatment increased the heteronymous EPSPs evoked by stimulation of the intact LGS nerve (Fig. $4 B$ ) by $33 \%$, but this slight increase was not significant $(0.1<p<0.2)$.

The NGF treatment was administered to 11 rats following crush of the MG nerve the day after birth. Unfortunately, however, only $15 \mathrm{MG}$ motoneurons were recorded from these animals. Thus, the lack of statistical significance of Figure $4 B$ might be attributed to the small sample size. From the same group of animals treated with NGF, 53 LGS motoneurons were recorded. Figure $5 A$ (solid-line histogram) shows the amplitude distribution of monosynaptic EPSPs evoked in LGS motoneurons
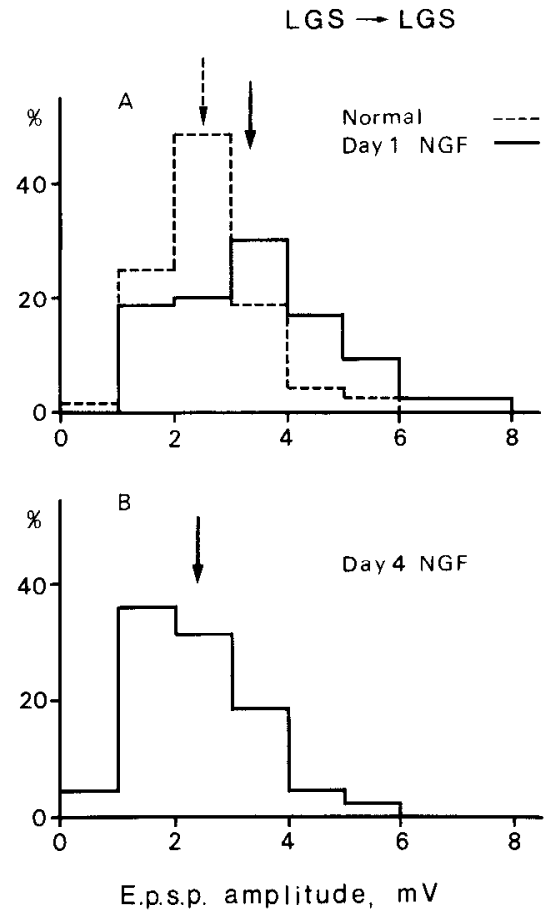

Figure 5. Effects of NGF on the homonymous EPSPs evoked by stimulation of the intact LGS nerve. The arrows indicate mean EPSP amplitudes. Their SDs and the numbers of motoneurons examined $(n)$ follow. $A$, Results obtained from 20 age-matched, unoperated rats (dashed-line histogram: $2.5 \pm 0.88 \mathrm{mV} ; n=144$ ) and those from 11 rats whose MG nerves had been crushed the day after birth and were treated with NGF $(3.3 \pm 1.4 \mathrm{mV} ; n=53)$. $B$, Results from 9 rats treated with NGF after the MG nerve crush made $4 \mathrm{~d}$ after birth $(2.3 \pm 0.90$ $\mathrm{mV} ; n=46$ ).

by stimulation of the intact LGS nerve in these NGF-treated animals. The mean EPSP amplitude was greater by $32 \%$ than that observed in age-matched, unoperated rats (dashed-line histogram). This difference was statistically significant $(p<0.001)$. Thus, the NGF treatment appears to enhance, slightly but significantly, the EPSPs elicited by stimulation of the intact LGS nerve. Again, however, the NGF treatment begun at $4 \mathrm{~d}$ of age failed to produce any significant effects $(p>0.2)$ on the homonymous EPSPs (Fig. 5B).

The above results may be summarized as follows: First, daily treatment with NGF significantly enhances monosynaptic EPSPs when the treatment is begun the day after birth, but not when it is begun after $4 \mathrm{~d}$ of age; second, the NGF effect is greater on the EPSPs evoked by stimulation of the crushed muscle nerve $(330-430 \%)$ than on those elicited by stimulation of the intact muscle nerve (32\%).

\section{Effects of anti-NGF antiserum on EPSPs}

The antiserum against NGF was applied to 8 rats from the day after birth, as described in Materials and Methods. The homonymous or heteronymous EPSPs recorded from MG or LGS motoneurons in these animals were not significantly different from those observed in age-matched normal rats (Kuno et al., 1985).

\section{Effects of NGF on motoneuron properties}

As shown in Figure 4, the EPSPs recorded from MG motoneurons in response to stimulation of the crushed $M G$ nerve were markedly enhanced by NGF treatment, whereas the NGF effect was marginal on the EPSPs evoked in the same motoneurons by stimulation of the intact LGS nerve. Therefore, EPSP am- 
Table 1. MG sensory neurons labeled in the DRG following unilateral crush of the MG nerve the day after birth

\begin{tabular}{llll} 
Animal & $\begin{array}{l}\text { Crushed side } \\
(\mathrm{A})\end{array}$ & $\begin{array}{l}\text { Control side } \\
(\mathrm{B})\end{array}$ & $\begin{array}{l}\mathrm{A} / \mathrm{B}(\%) \\
(\mathrm{C})\end{array}$ \\
\hline 1 & 89 & 153 & 58 \\
2 & 81 & 130 & 62 \\
3 & 86 & 164 & 52 \\
4 & 64 & 110 & 58 \\
5 & 102 & 188 & 54 \\
6 & 93 & 165 & 56 \\
7 & 63 & 103 & 61 \\
Mean \pm SD & $83 \pm 13$ & $145 \pm 29$ & $57 \pm 3$ \\
\hline
\end{tabular}

plitudes increased by NGF cannot be attributed to an increase in the input resistance of the motoneurons. Several electrophysiological properties of motoneurons, including resting and action potentials, input resistance, and amplitude and duration of afterhyperpolarization, were measured: These properties were not affected by treatment with NGF.

Recently, Schmalbruch (1984) reported that following crush of the sciatic nerve at birth, about $35 \%$ of the lumbar motoneurons die in the rat. In 5 rats, we counted MG motoneurons retrogradely labeled with HRP 35-45 d after unilateral crush of the MG nerve on the day after birth. An average loss of $28 \%$ of the MG motoneurons (range, 20-34\%) was found on the operated side compared to the contralateral, intact side. This deficit was statistically significant $(p<0.05)$. In 5 rats treated with NGF after the same operation, the loss of MG motoneurons on the operated side ( $42 \%$; range, $34-57 \%)$ was significantly $(p<$ $0.01)$ greater than that observed in the animals without NGF treatment. It is not clear how NGF treatment enhances the cell death of axotomized motoneurons.

\section{Sensory neuron death following nerve crush}

Table 1 shows the number of HRP-labeled DRG cells attached to the MG nerves on the side of crush performed on the day after birth (A) and on the control, intact side (B) in 7 animals. The difference between sides in the number of labeled cells, as tested by the matched-pairs $t$ test, was highly significant $(p<$ 0.001 ). Sensory neuron death following nerve crush was cstimated by the ratio of $A$ to $B$ (Table $1 C$ ). An average loss of $43 \%$ of $\mathrm{MG}$ sensory neurons was observed on the crushed side.

Table 2 shows the results obtained from animals whose $\mathrm{MG}$ nerves had been crushed unilaterally $4 \mathrm{~d}$ after birth. The difference between experimental and intact sides in the number of labeled cells was not significant $(0.10<p<0.20)$.

Effects of NGF on cell death induced by nerve crush

The data shown in Table 3 were obtained from animals that received NGF treatment following unilateral MG nerve crush

$\begin{aligned} & \text { Table 2. MG sensory neurons labeled in the DRG following } \\
& \text { unilateral crush of the MG nerve } \mathbf{4} \mathbf{d} \text { after birth }\end{aligned}$
\begin{tabular}{llll} 
Animal & Crushed side & $\begin{array}{l}\text { Control side } \\
\text { (B) }\end{array}$ & $\begin{array}{l}\text { A/B (\%) } \\
\text { (C) }\end{array}$ \\
\hline 1 & 130 & 126 & 103 \\
2 & 104 & 143 & 73 \\
3 & 179 & 214 & 84 \\
4 & 181 & 173 & 105 \\
Mean \pm SD & $149 \pm 33$ & $164 \pm 33$ & $91 \pm 13$ \\
\hline
\end{tabular}
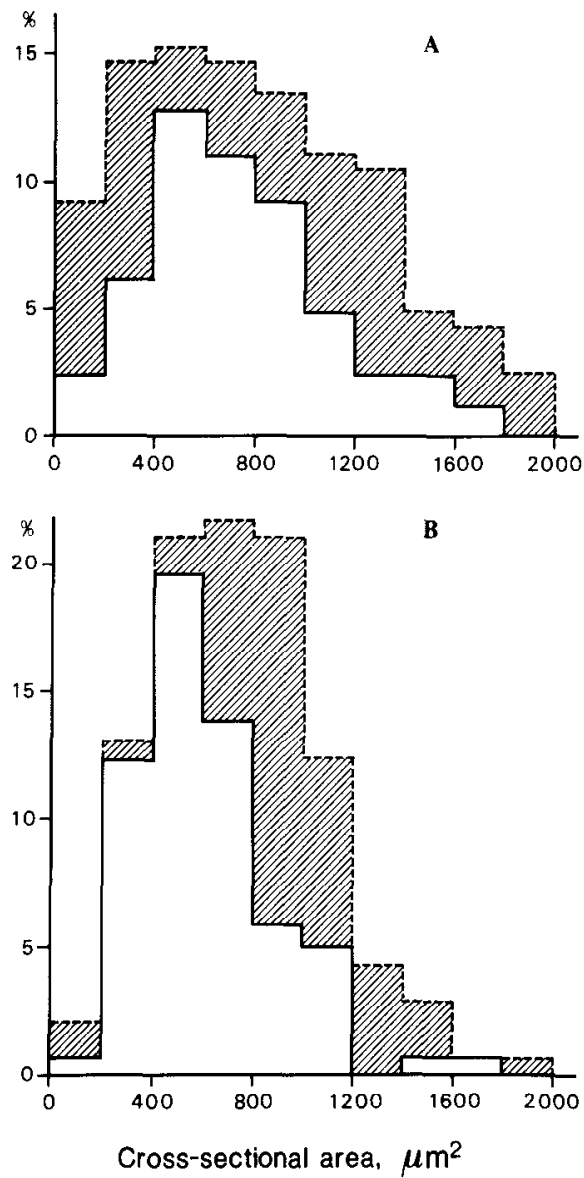

Figure 6. Size spectra of MG sensory neurons on the intact (dashedline histograms) and nerve-crushed (solid-line histograms) sides. The MG nerve was unilaterally crushed on the day after birth. $A$, Without NGF treatment. $B$, With NGF treatment. Abscissas, Cross-sectional areas of MG DRG cells. Ordinates, Percentages relative to the total MG sensory neurons measured on the intact side. Shaded areas indicate cell deficits through nerve crush.

on the day after birth. The difference in the number of labeled cells between the experimental and intact sides was significant $(p<0.02)$. On the average, a $36 \%$ loss of MG sensory neurons was observed on the crushed side in these NGF-treated animals (Table 3C). The difference in cell loss between the NGF-treated (Table 3) and NGF-untreated (Table 1) groups was statistically examined by $t$ test or by more sensitive tests, including the Wilcoxon test and the Mann-Whitney $U$ test. None of these tests revealed any significant difference between the 2 groups.

Table 3. NGF effects on the number of MG sensory neurons labeled in the DRG following unilateral crush of the MG nerve the day after birth

\begin{tabular}{llll} 
Animal & $\begin{array}{l}\text { Crushed side } \\
\text { (A) }\end{array}$ & $\begin{array}{l}\text { Control side } \\
(\mathrm{B})\end{array}$ & $\begin{array}{l}\text { A/B (\%) } \\
\text { (C) }\end{array}$ \\
\hline 1 & 148 & 162 & 91 \\
2 & 68 & 145 & 47 \\
3 & 94 & 185 & 51 \\
4 & 88 & 189 & 47 \\
5 & 87 & 152 & 57 \\
6 & 184 & 200 & 92 \\
Mean \pm SD & $112 \pm 41$ & $172 \pm 20$ & $64 \pm 20$ \\
\hline
\end{tabular}


We further examined whether a particular group of sensory neurons is more likely to die following nerve crush, with or without NGF treatment. For this purpose, the cross-sectional areas of all HRP-labeled DRG cclls were measured and used as a criterion for cell size (Harper and Lawson, 1985). Figure $6 \mathrm{~A}$ shows the size-frequency histograms of labeled MG sensory cells on the control (dashed-line histogram) and experimental (solid-line histogram) sides in an animal whose MG nerve was unilaterally crushed the day after birth (Animal 5 in Table 1). It can be seen that cell death induced by nerve crush occurs uniformly over the entire size spectrum (shaded area). Figure $6 B$ shows similar histograms obtained from an animal whose MG nerve was unilaterally crushed on day 1 , and immediately treated with NGF (Animal 5 in Table 3). A deficit of MG sensory cells on the operated side (solid-line histogram) is evident when compared to the control side (dashed-line histogram). However, cell loss was predominantly observed in large sensory neurons (shaded area). Harper and Lawson (1985) have shown that in the rat $\mathrm{DRG}, \mathrm{A} \delta$, and $\mathrm{C}$ cells (axonal velocity $<8 \mathrm{~m} / \mathrm{sec}$ ) have cross-sectional areas of less than $900 \mu \mathrm{m}^{2}$, whereas $\mathrm{A} \alpha$ cells (axonal velocity $>30 \mathrm{~m} / \mathrm{sec}$ ) have larger cross-sectional areas $\left(800-2000 \mu \mathrm{m}^{2}\right)$. Since these data may not apply to the 4-5week-old rats used in our study, the labeled MG sensory cells were arbitrarily divided into 2 groups by cross-sectional areas, below and above $600 \mu \mathrm{m}^{2}$. The cell deficit of the large neuron group in NGF-treated animals $(67 \% ; n=6)$ was not significantly different $(0.2<p<0.3)$ from that observed following nerve crush alone $(56 \% ; n=7)$. In contrast, the NGF-treated rats showed a significantly $(p<0.05)$ smaller deficit in the small neuron group (1\%) than those without NGF treatment $(33 \%)$. This suggests that cell death of $\mathrm{A} \delta$ and $\mathrm{C}$ sensory neurons induced by peripheral nerve crush can be prevented by NGF treatment, whereas this treatment is ineffective in preventing cell death of $A \alpha$ sensory neurons. The extent of cell death observed following nerve crush was significantly $(p<0.005)$ greater in the large neuron group (56\%) than in the small group (33\%). This might account for the lack of a significant difference in the total deficit of sensory neurons between NGF-treated and NGFuntreated animals (Tables 1 and 3).

\section{Discussion}

Consistent with recent reports (Bondok and Sansone, 1984; Yip and Johnson, 1984; Yip et al., 1984), approximately 50\% of the sensory neurons were found to die when the peripheral axons were crushed on the day after birth. Yip et al. (1984) have shown that sensory neuron death induced by crush of the sciatic nerve in newborn rats begins to occur within $12 \mathrm{hr}$ and is complete within $24 \mathrm{hr}$. NGF treatment has been reported to retard the onset of sensory cell death induced by axotomy (Yip and Johnson, 1984; Yip et al., 1984), but fails to maintain the survival of the sensory neurons that are destined to die (Yip et al., 1984). Thus, when the DRG was examined 3 weeks after treatment with NGF following peripheral axotomy at birth, the cell deficit was the same as that following the axotomy (Yip et al., 1984). In our results, the loss of large sensory neurons examined 25$35 \mathrm{~d}$ after peripheral axotomy was also unaffected by NGF treatment. However, the cell death of small sensory neurons (presumably $\mathrm{A} \delta$ and $\mathrm{C}$ cells) was significantly prevented by exogenous NGF (Fig. 6). Exposure to anti-NGF antibodies or antiserum is also known to result in a preferential loss of small sensory neurons (Johnson et al., 1980; Yip et al., 1984).

The degree of depression of the EPSPs evoked by stimulation of the MG nerve crushed on day $1(87-91 \%)$ or day $4(45 \%)$ was greater than that expected from the loss of sensory cells following nerve crush on day $1(43 \%)$ or day 4 (insignificant). Therefore, synaptic depression must occur at those synapses formed by the surviving sensory neurons whose axons had previously been crushed. Since NGF treatment did not prevent the cell death of large Group Ia sensory neurons (A $\alpha$ cells), an increase in the EPSP amplitude by NGF can be attributed to a partial reversal of the depression at the synapses formed by the axotomized sensory fibers. NGF treatment did enhance the EPSPs elicited by stimulation of the intact muscle nerve. However, this effect was marginal compared to increased EPSPs evoked by stimulation of the crushed nerve. Thus, enhancement of the EPSPs by NGF depends upon the state of the sensory neurons. Therefore, it is likely that the site of action of exogenous NGF is at the presynaptic level. NGF treatment has been shown to cause the formation of de novo muscle spindles in reinnervated muscles of neonatal rats as a result of increased sprouting of peripheral sensory collaterals (Sekiya et al., 1986). It is possible that similar sprouting may occur in central terminals of the sensory neurons through NGF treatment.

NGF was effective in increasing EPSPs when treatment began on the day after birth, but was ineffective when the treatment began $4 \mathrm{~d}$ after birth. Thus, sensory neurons appear to be more responsive to NGF when the neurons are less differentiated. This may account for the observation that small sensory neurons (A $\delta$ and $\mathrm{C}$ cells) are more responsive to NGF than are large neurons (A $\alpha$ cells), since the small neurons are born later in gestation (Lawson et al., 1974). Huizar et al. (1975) have suggested that axotomy may lead to "dedifferentiation" of neuron properties. Under such conditions, the sensory neurons would become more responsive to NGF. The greater effect of NGF on the EPSPs evoked by stimulation of the axotomized MG nerve than on those elicited from the intact nerve may be accounted for on this basis.

We have no adequate explanation for the observation that the antiserum against NGF fails to produce any significant effects on EPSPs. The administration of the NGF antiserum to newborn rats causes primarily a loss of small sensory neurons (Yip et al., 1984). Thus, Group Ia sensory neurons may be more resistant to NGF deprivation. Alternatively, the antiserum may be unable to cross the capsule surrounding muscle spindles, so that Group Ia fibers may be maintained by NGF that is possibly present within the capsule (Kuno et al., 1985). Whatever the explanation, the present results provide no evidence that the central synaptic function of Group Ia sensory neurons is normally maintained by endogenous NGF. However, the present results do show that small sensory neurons are not the only sensory neurons responsive to exogenous NGF in postnatal life.

Early studies on the DRG of the chick embryo (Levi-Montalcini and Hamburger, 1951) suggested that only the dorsomedial group of cells is responsive to NGF. However, both dorsomedial and ventrolatcral cells are now known to be dependent upon NGF for their survival and differentiation in embryos (Hamburger et al., 1981). Both cell groups contain proprioceptive and exteroceptive sensory neurons (Honig, 1982). It is likely that all sensory neurons are responsive to NGF in postnatal life, as well as at the embryonic stage. However, it should be noted that factors other than NGF may also be involved in the maintenance of sensory neurons (Richardson and Ebendal, 1982; Thoenen and Barde, 1980).

A puzzling phenomenon was the enhanced cell death of axotomized motoneurons by NGF treatment. One might speculate that, because of increased loss of MG motoneurons, the remaining $\mathrm{MG}$ motoneurons may receive synaptic inputs from a larger number of Group Ia fibers as a consequence of sprouting and synaptic rearrangements following NGF treatment. However, the small increase of motoneuron death (about 15\%) cannot entirely account for the three- to fourfold increase in the mean EPSP amplitude observed following treatment with NGF.

\section{References}

Bocchini, V., and P. U. Angeletti (1969) The nerve growth factor: Purification as a 30,000-molecular-weight protein. Proc. Natl. Acad. Sci. USA 64: 787-794. 
Bondok, A. A., and F. M. Sansone (1984) Retrograde and transganglionic degeneration of sensory neurons after a peripheral nerve lesion at birth. Exp. Neurol. 86: 322-330.

Burke, R. E. (1968) Group Ia synaptic input to fast and slow twitch motor units of cat triceps surae. J. Physiol. (Lond.) 196: 605-630.

Csillik, B., M. E. Schwab, and H. Thoenen (1985) Transganglionic regulation of central terminals of dorsal root ganglion cells by nerve growth factor (NGF). Brain Res. 331: 11-15.

Eccles, J. C. (1957) The Physiology of Nerve Cells, Johns Hopkins U.P., Baltimore, MD.

Eccles, J. C., K. Krnjević, and R. Miledi (1959) Delayed effects of periphcral scverance of afferent nerve fibres on the efficiency of their central synapses. J. Physiol. (Lond.) 145: 204-220.

Fenton, E. L. (1970) Tissue culture assay of nerve growth factor and of the specific antiserum. Exp. Cell Res. 59: 383-392.

Fitzgerald, M., P. D. Wall, M. Goedert, and P. C. Emson (1985) Nerve growth factor counteracts the neurophysiological and neurochemical effects of chronic sciatic nerve section. Brain Res. 332: 131-141.

Gallego, R., M. Kuno, R. Núñez, and W. D. Snider (1979) Disuse enhances synaptic efficacy in spinal motoneurones. J. Physiol. (Lond.) 291: 191-205.

Gallego, R., M. Kuno, R. Núñez, and W. D. Snider (1980) Enhancement of synaptic function in cat motoneurones during peripheral sensory regeneration. J. Physiol. (Lond.) 306: 205-218.

Goedert, M., K. Stoeckel, and U. Otten (1981) Biological importance of the retrograde axonal transport of nerve growth factor in sensory neurons. Proc. Natl. Acad. Sci. USA 78: 5895-5898.

Goldring, J. M., M. Kuno, R. Núñez, and W. D. Snider (1980) Reaction of synapses on motoneurones to section and restoration of peripheral sensory connexions in the cat. J. Physiol. (Lond.) 309: 185198.

Hamburger, V., J. K. Brunso-Bechtold, and J. W. Yip (1981) Neuronal death in the spinal ganglia of the chick embryo and its reduction by nerve growth factor. J. Neurosci. 1: 60-71.

Hamm, T. M., B. R. Botterman, R. M. Reinking, and D. G. Stuart (1983) Characteristics of $M$ spikes in cat motoneurons and their significance for the measurement of small composite la EPSPs. Exp. Brain Res. 49: 68-76.

Harper, A. A., and S. N. Lawson (1985) Conduction velocity is related to morphological cell type in rat dorsal root ganglion neurones. J. Physiol. (Lond.) 359: 31-46.

Honig, M. G. (1982) The development of sensory projection patterns in embryonic chick hind limb. J. Physiol. (Lond.) 330: 175-202.

Huizar, P., M. Kuno, and Y. Miyata (1975) Differentiation of motoneurones and skeletal muscles in kittens. J. Physiol. (Lond.) 252 . $465-479$.

Johnson, E. M., P. D. Gorin, L. D. Brandeis, and J. Pearson (1980)
Dorsal root ganglion neurons are destroyed by exposure in utero to maternal antibody to nerve growth factor. Science 210: 916-918.

Kessler, J. A., and I. B. Black (1980) Nerve growth factor stimulates the development of substance $P$ in sensory ganglia. Proc. Natl. Acad. Sci. USA 77: 649-652.

Kornblum, H. I., and E. M. Johnson (1982) Time and dose dependencies of effects of nerve growth factor on sympathetic and sensory neurons in neonatal rats. Brain Res. 234: 41-51.

Kuno, M., Y. Miyata, S. Homma, and M. Ogawa (1985) Nerve growth factor enhances central synaptic function of Ia sensory neurons. Neurosci. Res. 2: 275-280.

Lawson, S. N., K. W. T. Caddy, and T. J. Biscoe (1974) Development of rat dorsal root ganglion neurones: Studies of cell birthdays and changes in mean cell diameter. Cell Tissue Res. 153: 399-413.

Levi-Montalcini, R., and V. Hamburger (1951) Selective growth-stimulating effects of mouse sarcoma on the sensory and sympathetic nervous system of the chick embryo. J. Exp. Zool. 116: 321-362.

Otten, U., M. Goedert, N. Mayer, and F. Lembeck (1980) Requirement of nerve growth factor for development of substance P-containing sensory neurones. Nature 287: 158-159.

Richardson, P. M., and T. Ebendal (1982) Nerve growth activities in rat peripheral nerve. Brain Res. 256: 57-64.

Schmalbruch, H. (1984) Motoneuron death after sciatic nerve section in newborn rats. J. Comp. Neurol. 224: 252-258.

Sekiya, S., S. Homma, Y. Miyata, and M. Kuno (1986) Effects of nerve growth factor on differentiation of muscle spindles following nerve lesion in neonatal rats. J. Neurosci. 6: 2019-2025.

Stöckel, K., M. Schwab, and H. Thoenen (1975a) Specificity of retrograde transport of nerve growth factor (NGF) in sensory ncurons: A biochemical and morphological study. Brain Res. 89: 1-14.

Stöckel, K., M. Schwab, and H. Thoenen (1975b) Comparison between the retrograde axonal transport of nerve growth factor and tetanus toxin in motor, sensory and adrenergic neurons. Brain Res. 99: 1-16.

Streit, P., and J. C. Reubi (1977) A new and sensitive staining method for axonally transported horseradish peroxidase (HRP) in the pigeon visual system. Brain Res. 126: 530-537.

Thoenen, H., and Y.-A. Barde (1980) Physiology of nerve growth factor. Physiol. Rev. 60: 1284-1335.

Yip, H. K., and E. M. Johnson (1984) Developing dorsal root ganglion neurons require trophic support from their central processes: Evidence for a role of retrogradely transported nerve growth factor from the central nervous system to the periphery. Proc. Natl. Acad. Sci. USA 81: 6245-6249.

Yip, H. K., M. Rich, P. A. Lampe, and E. M. Johnson (1984) The effects of nerve growth factor and its antiserum on the postnatal development and survival after injury of sensory neurons in rat dorsal root ganglia. J. Neurosci. 4: 2986-2992. 